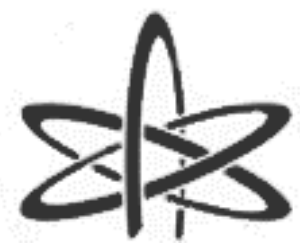

BJRS
BRAZILIAN JOURNAL

$\mathrm{OF}$

RADIATION SCIENCES

08-01A (2020) 01-06

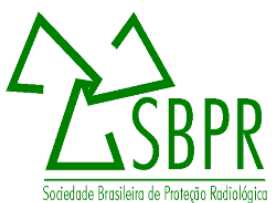

\title{
Some characterization tests for an extrapolation chamber in CT standard beams in a specific chamber depth
}

\author{
M. C. Castro; N. F. Silva; L. V. E. Caldas \\ Instituto de Pesquisas Energéticas e Nucleares, Comissão Nacional de Energia Nuclear (IPEN / CNEN - SP), \\ Av. Professor Lineu Prestes 2242, 05508-000, São Paulo, SP, Brazil \\ maysadecastro@gmail.com
}

\begin{abstract}
Among all diagnostic exams, the computed tomography (CT) is responsible for the highest dose values to the patients. So, the radiation doses in this procedure must be accurate for the dosimetry procedure in CT scanner beams making use of pencil ionization chambers with sensitive volume lengths of $10 \mathrm{~cm}$. However, there is no primary standard system for this kind of radiation beam. An extrapolation ionization chamber, built at the Calibration Laboratory (LCI) was tested to establish a CT primary standard. The aim of this work was to perform some characterization tests (short- and medium-term stabilities, energy dependence, angular dependence) in the standard X-ray beams established for computed tomography at the LCI in a specific chamber depth (1.25 mm). The results showed to be within the international recommended limits except for the energy dependence.
\end{abstract}

Keywords: Extrapolation chamber, computed tomography, dosimetry. 


\section{INTRODUCTION}

The use of Computed Tomography (CT) for diagnostic images has been growing due to technological advances of this equipment [1-3]. Therefore, there is a concern regarding the dose received by the patients undergoing this procedure, because it uses higher radiation doses when compared to other fields of conventional radiology, and this procedure needs to be performed with the highest precision and accuracy possible.

For the dosimetry of CT beams, the radiation detector is usually a pencil-type ionization chamber. It presents a uniform response to the incident radiation beam for all angles. However, there is no primary standard system for this kind of radiation beam. A homemade extrapolation chamber was tested in this work to establish a CT primary standard for calibration laboratory beams $[4,5]$.

The extrapolation chamber is a parallel-plate ionization chamber which enables the variation of the distance between the collecting electrode and the entrance window, allowing different sensitive air volumes. This kind of ionization chamber is mostly utilized in beta radiation dosimetry [4], and it was tested for low-energy radiation beams too, showing results within the internationally acceptable limits [5-7].

This work had the objective to study the response stability of an extrapolation chamber, as well as obtaining the energy and angular dependence in standard CT beams of a calibration laboratory.

\section{MATERIALS AND METHODS}

The extrapolation chamber tested in this work has a collecting electrode of $30 \mathrm{~mm}$ in diameter, the entrance window is made of aluminized polyethylene terephthalate with density of $0.84 \mathrm{mg} / \mathrm{cm}^{2}$, and the collecting electrode and guard ring are made of graphite; this chamber was developed at the Calibration Laboratory of Instruments / IPEN (LCI) [6].

For the short and medium-term stability study of the extrapolation chamber response, a ${ }^{90} \mathrm{Sr}+{ }^{90} \mathrm{Y}$ control source $(33 \mathrm{MBq})$ was utilized. In order to obtain the energy dependence and the angle dependence of the extrapolation chamber response, an X-ray system Pantak/Seifert (ISO- 
VOLT model $160 \mathrm{HS}$ ) was utilized, which operates up to $160 \mathrm{kV}$. Table 1 presents the CT radiation qualities at the LCI.

Table 1: Characteristics of the CT standard X radiation qualities at the LCI, based on the Report IEC 61267 [8].

\begin{tabular}{ccccc}
\hline $\begin{array}{c}\text { Radiation } \\
\text { Quality }\end{array}$ & $\begin{array}{c}\text { Tube Voltage } \\
(\mathbf{k V})\end{array}$ & $\begin{array}{c}\text { Tube Current } \\
(\mathbf{m A})\end{array}$ & $\begin{array}{c}\text { HVL } \\
(\mathbf{m m A l})\end{array}$ & $\begin{array}{c}\text { Air Kerma Rate } \\
(\mathbf{m G y} / \mathbf{m i n})\end{array}$ \\
\hline RQT 8 & 100 & 10 & 6.9 & 22.0 \\
RQT 9 $\dagger$ & 120 & 10 & 8.4 & 34.0 \\
RQT 10 & 150 & 10 & 10.1 & 57.0 \\
\hline
\end{tabular}

$\dagger$ Reference CT radiation quality at LCI/IPEN

HVL: Half-Value Layer

The uncertainties of type A and type B were determined, with the combined uncertainty of a coverage factor $\mathrm{k}=2$.

\section{RESULTS AND DISCUSSION}

Initially the results for the short- and medium-term stability tests for the extrapolation chamber are presented. Ten consecutive measurements were taken each time during a period of 4 months. All of the mean values were corrected for the standard environmental conditions. For the short-term stability test (diagnostic radiology qualities) of the extrapolation chamber, the standard deviation must not exceed $1 \%$. For the medium-term stability test the results obtained for the short-term stability test on different days were considered, and the standard deviation should not exceed $2 \%$ [8].

Figure 1 shows the response normalized to the mean value of the first ten measurements of the extrapolation chamber. The result for the short-term stability test was $0.04 \%$, in accordance with IEC 61674 [8]. 
Figure 1: Medium-term stability of the extrapolation chamber response; the maximum uncertainty of the measurements was $0.61 \%$, not visible in the figure.

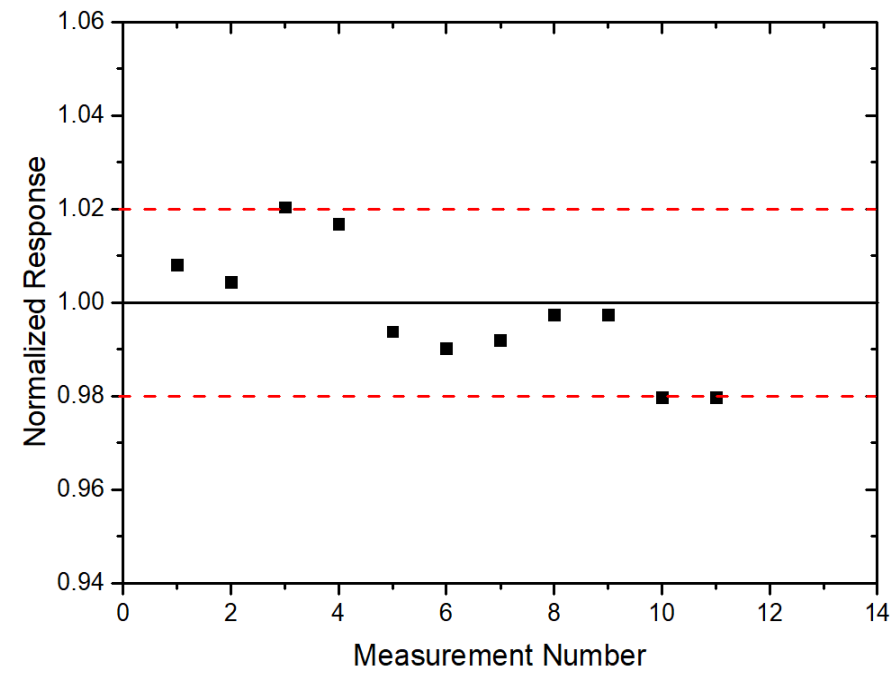

As observed in Figure 1, the medium-term stability of the extrapolation chamber response is within the recommended limits [8].

For the energy dependence, ten consecutive measurements were taken each time, and all of them were corrected according to the standard environmental conditions too. To analyze the energy dependence of the extrapolation chamber response for different radiation qualities, the results were compared with those of the reference radiation quality (RQT 9). The calibration coefficients and the correction factors were obtained, and they must not exceed $5 \%$ [8]. Table 2 shows the energy dependence for the extrapolation chamber response in CT radiation qualities.

Table 2: Energy dependence of the extrapolation chamber response.

\begin{tabular}{lccc}
\hline $\begin{array}{c}\text { Radiation } \\
\text { Quality }\end{array}$ & $\begin{array}{c}\text { Calibration Coefficient } \\
(\mathbf{m G y} / \mathbf{p C})\end{array}$ & $\begin{array}{c}\text { Correction } \\
\text { Factor }\end{array}$ & $\begin{array}{c}\text { Deviation of the } \\
\text { Energy Dependence } \\
(\%)\end{array}$ \\
\hline RQT 8 & $0.0200 \pm 0.0003$ & $0.935 \pm 0.001$ & 6.5 \\
RQT 9 & $0.0214 \pm 0.0003$ & $1.000 \pm 0.001$ & --- \\
RQT 10 & $0.0229 \pm 0.0003$ & $1.072 \pm 0.001$ & 7.2 \\
\hline
\end{tabular}


As can be observed in Table 2, the energy dependence is not within the internationally acceptable limits [8], but these results were expected, because the sensitive volumes and the geometries of the extrapolation chamber and the pencil-type ionization chamber are very different.

The angular dependence was obtained for the radiation qualities for computed tomography beams; ten measurements were taken for each incident radiation angle. The extrapolation chamber was rotated to both sides, and the results obtained were normalized to the initial position (centralized extrapolation chamber location). Following the recommendation based on the report IEC 61674, the standard deviation should not exceed $3 \%$. Figure 2 shows the results obtained for the angular dependence.

Figure 2: Angular dependence of the extrapolation chamber response; the maximum uncertainty of the measurements was $0.04 \%$, for the radiation quality RQT 10 .

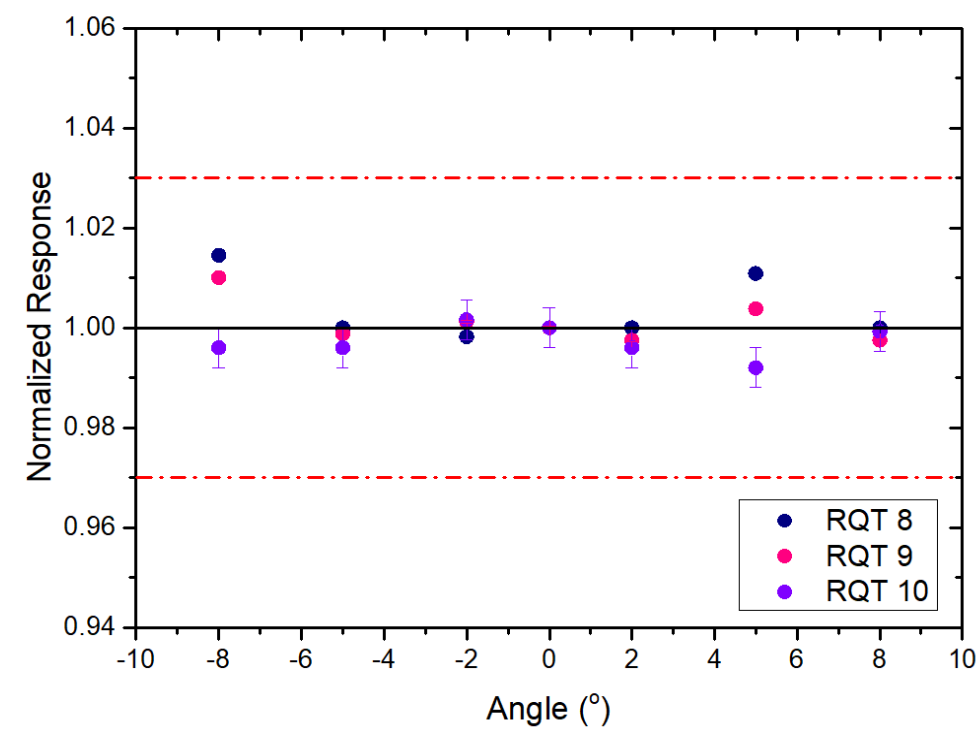

The results obtained for the angular dependence study of the extrapolation chamber response are within the recommended limits [8].

\section{CONCLUSION}

The results obtained for four characterization tests of the homemade extrapolation chamber at a specific chamber depth $(1.25 \mathrm{~mm})$ in standard computed tomography beams were in agreement with 
the international recommendations, except for the energy dependence. The decision about the extrapolation chamber depth for the graphite collecting electrode was appropriate, and in the future it will be possible to use this same chamber depth with an aluminum collecting electrode.

\section{ACKNOWLEDGMENTS}

The authors acknowledge the partial financial support from the Brazilian agencies: CNEN, CNPq (Project 301335/2016-8), CAPES (Project 554/2018).

\section{REFERENCES}

[1] BOONE, J. M. The trouble with CTDI100. Med. Phys. 34(4) 1364-1371, 2007.

[2] McCOLLOUGH, C. H. Computed tomography technology and dose in the 21 st century. Health Physics, 116(2), 157-162, 2019.

[3] SMITH-BINDMAN, R., WANG, Y., CHU, P., et al. International variation in radiation dose for computed tomography examinations: prospective cohort study. BMJ, 1-12, 2019.

[4] DIAS, S.K.; CALDAS, L V E. Development of an extrapolation chamber for the calibration of beta-ray applicators. IEEE Trans. Nucl. Sci. 45, 1666-1669, 1998.

[5] DIAS, S.K.; CALDAS, L V E. Extrapolation chamber response in low-energy radiation standard beams. J. Appl. Phys. 89, 669-671, 2001.

[6] NEVES, L P; SILVA, E A B; PERINI, A P; MAIADANA, N L; CALDAS, L V E. Characterization of an extrapolation chamber for low-energy X-rays: Experimental and Monte Carlo preliminary results. Appl. Radiat. Isot. 70, 1388-1391, 2012.

[7] FIGUEIREDO, M.T.T; SILVA, T.A. Determination of the effective volume of an extrapolation chamber for X-ray dosimetry, In: X CONGRESO REGIONAL LATINOAMERICANO IRPA DE PROTECTIÓN Y SEGURIDAD RADIOLÓGICA, 2015, Buenos Aires. Annals 2015.

[8] IEC - International Electrotechnical Commission. Medical Diagnostic X-ray Equipment. Radiation Conditions for Use in the Determination of Characteristics. IEC 61267. 2nd ed. Genève IEC, 2005. 